\section{Pflicht zur Fortbildung vor Reha-Verordnung fällt weg}

_ Die Vereinbarung von Qualitätssicherungsmaßnahmen zur Verordnung von Leistungen zur medizinischen Rehabilitation auf dem Muster 61 wird außer Kraft gesetzt. Hintergrund ist, dass zum 1. April 2016 die Rehabilitations-Richtlinie des Gemeinsamen Bundesausschusses (G-BA) geändert wurde und Vertragsärzte nun keine besonderen Qualifikationen mehr brauchen.

Bisher mussten Hausärzte z.B. die fakultative Weiterbildung „Klinische
Geriatrie“ absolvieren, ein Jahr in einer Rehabilitationseinrichtung arbeiten oder eine Fortbildung von 16 Stunden über Grundlagen der International Classification of Functioning, Disability and Health (ICF) und die verordnungsfähigen Reha-Leistungen absolvieren.

\section{MMW-KOMMENTAR}

Die Qualitätssicherungsvereinbarung war seinerzeit beschlossen worden, um die Qualifikationserfordernisse konkret auszugestal-

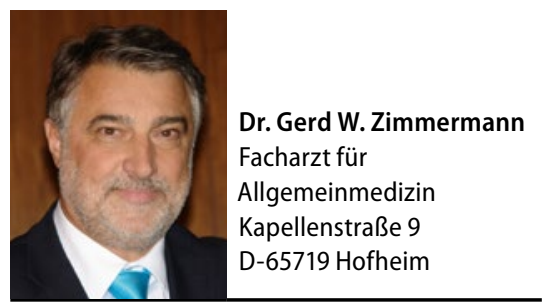

ten. Sie definiert vor allem die Inhalte des Fortbildungskurses sowie die Anforderungen an den Kursleiter und die Referenten. Mit der Änderung der Rehabilitations-Richtlinie sind die Inhalte der Vereinbarung überflüssig geworden. Aus diesem Grund haben sich KBV und Krankenkassen auf eine Aussetzung zum 1. April 2016 geeinigt. Aber Achtung: Die Verordnung von Reha-Maßnahmen zulasten anderer Versicherungsträger - z. B. der Rentenversicherung - ist von dieser Neuerung nicht betroffen.

\title{
Geriatrische Versorgung wird ab Juli gestärkt
}

Tab. 1 Neue Geriatrie-Ziffern ab

1. Juli 2016 (für Hausärzte in blau)

\begin{tabular}{|l|l|l|}
\hline EBM & \multicolumn{2}{|l|}{ Legende und Punkte } \\
\hline 03360 & $\begin{array}{l}\text { Hausärztlich-geriatrisches } \\
\text { Basisassessment }\end{array}$ & 122 \\
\hline 03362 & $\begin{array}{l}\text { Hausärztlich-geriatrischer } \\
\text { Betreuungskomplex }\end{array}$ & 159 \\
\hline 30984 & $\begin{array}{l}\text { Weiterführendes geriatri- } \\
\text { sches Assessment }\end{array}$ & 882 \\
\hline 30985 & $\begin{array}{l}\text { Zuschlag zur Nr. 30984 für } \\
\text { weitere 30 Minuten }\end{array}$ & 325 \\
\hline 30986 & $\begin{array}{l}\text { Zuschlag zur Nr. 30985 je } \\
\text { weitere 30 Minuten }\end{array}$ & 234 \\
\hline 30980 & $\begin{array}{l}\text { Abklärung vor Erbrin- } \\
\text { gung der Nr. 30984 }\end{array}$ & 194 \\
\hline 30981 & $\begin{array}{l}\text { Abklärung vor Erbrin- } \\
\text { gung der Nr. 30984 }\end{array}$ & 131 \\
\hline 30988 & $\begin{array}{l}\text { Zuschlag zur Nr. 03362 } \\
\text { (nur nach Nr. 30984) }\end{array}$ & 65 \\
\hline & & \\
\hline
\end{tabular}

ist ein „weiterführendes“ geriatrisches Assessment nach Nr. 30984. Dieses kann aber nur von Fachärzten für Innere Medizin und Geriatrie, Internisten mit dem Schwerpunkt Geriatrie oder Ärzten mit der Zusatzbezeichnung Geriatrie berechnet werden.

Im Hausarzt-Kapitel bleibt es bei den gewohnten Geriatrieleistungen, also dem Basisassessment nach Nr. 03360 (122 Punkte, 12,73 Euro) und dem Betreuungskomplex nach Nr. 03362 (159 Punkte, 16,59 Euro). Neu ist, dass man den Betreuungskomplex auch abrechnen darf, wenn man nicht selbst das Basisassessment erhoben hat, sondern den $\mathrm{Pa}$ tienten für das weiterführende Assessment an den Geriater überwiesen hat.

Wenn der Hausarzt den Patienten überweisen will, muss die Notwendigkeit zunächst gemeinsam mit dem Geriater konsiliarisch abgeklärt werden. Für diese Abklärung berechnet der Hausarzt die neue Nr. 30980 (einmal im Krank- heitsfall, 194 Punkte), der Geriater die Nr. 30981. Letzterer führt dann das Assessment nach Nr. 30984 durch. Im Anschluss ist der Hausarzt berechtigt, die Nr. 30988 (65 Punkte) als Zuschlag zum geriatrischen Betreuungskomplex nach Nr. 03362 abzurechnen. Das muss allerdings binnen vier Wochen nach dem Besuch beim Geriater geschehen.

\section{MMW-KOMMENTAR}

Wer selbst das weiterführende Assessment anbieten möchte, muss die Zusatzbezeichnung "Geriatrie" erwerben. Die Voraussetzungen sind regional unterschiedlich. Grundlage ist eine Weiterbildung von 18 Monaten bei einem Weiterbildungsermächtigten. In Berlin müssen es nur sechs Monate sein, wobei vierwöchige Abschnitte anrechenbar sind. Die restliche Qualifikation kann in einem 120-Stunden-Kurs erworben werden. Die Vergütung der neuen Betreuungsleistungen soll extrabudgetär erfolgen, wobei die Krankenkassen noch zustimmen müssen. 